\title{
On Recovering from Run-time Misbehaviour in ADR*
}

\author{
Kyriakos Poyias Emilio Tuosto \\ Department of Computer Science, University of Leicester, UK \\ kyriakospoyias@gmail.com emilio@le.ac.uk
}

\begin{abstract}
We propose a monitoring mechanism for recording the evolution of systems after certain computations, maintaining the history in a tree-like structure. Technically, we develop the monitoring mechanism in a variant of ADR (after Architectural Design Rewriting), a rule-based formal framework for modelling the evolution of architectures of systems.

The hierarchical nature of ADR allows us to take full advantage of the tree-like structure of the monitoring mechanism. We exploit this mechanism to formally define new rewriting mechanisms for ADR reconfiguration rules. Also, by monitoring the evolution we propose a way of identifying which part of a system has been affected when unexpected run-time behaviours emerge. Moreover, we propose a methodology to suggest reconfigurations that could potentially lead the system in a non-erroneous state.
\end{abstract}

\section{Introduction}

We present a technical development of the Architectural Design Rewriting model (ADR) [4] that combines the features of ADR described in [4, 11]. We take the motivation of our work from the problems arising in modern software development. Software systems are no longer static and become more and more dynamic; because of their very interactive nature, such systems are starting to be studied under new angles [6]. For instance, software needs to adapt to the (often unpredictable) changes of the (virtual and physical) environment it operates in. The term autonomic computing has been coined to mark such systems [7], which present new degrees of complexity since they require high levels of flexibility and adaptiveness [8].

Such complexity calls for rigorous methods at very early stages of software development. Architectural Description Languages (ADLs) used to design such systems have to be able to guarantee software quality and correctness by being flexible to adapt from their initial designs, and also be able to predict the possible problems that could arise during the execution of such systems. Formal approaches aim to devise robust engineering practices to form reliable software products to mitigate the issues described above. Arguably, those approaches focus on software behaviour; correctness and efficiency of software play in fact a crucial role not only in critical systems but also in daily-life applications. In the design phase, semi-formal methods are typically adopted; as an example, the use of modelling languages is combined with design patterns to devise a model that can be checked. This approach may involve formal techniques (e.g., type or model checking) to guarantee properties of applications while non-formal techniques (or tools not supported by formal approaches) are typically used to tackle architectural design aspects. Our research agenda envisages the combination of those approaches with techniques to address the issues above at the design level. We believe that a rigorous treatment at the design level would allow to identify and solve many problems that are currently tackled only by inspecting or testing code.

We describe a formal framework that is able to tackle the architectural/structural aspects of the design and allow designers to identify and address problems at a higher level. In [11] we advocate a

${ }^{*}$ This work has been supported by FP7-PEOPLE-2011-IRSES MEALS

M. Carbone, I. Lanese, A. Lluch-Lafuente, A. Sokolova (Eds.):

6th Interaction and Concurrency Experience (ICE 2013)

EPTCS 131, 2013, pp. 68-84 doi 10.4204/EPTCS.131.7 
design-by-contract (DbC approach) for ADLs that allows the specification of contracts that architectures have to abide by. On these grounds, in [11] we propose a methodology that is able to compute structural "rearrangements" of systems' architecture to adapt themselves when an erroneous state is reached. Technically, the DbC approach in [11] is developed by extending ADR [4] with asserted production rules, that is rules for architectural transformations equipped with logical pre- and post-conditions. In this way, asserted productions become contracts that guarantee the architectural style when they are applied. The concept of architectural style is crucial in software architectures [12]. In ADR, the architectural style of a system is formalised in terms of productions rules, namely rules that can be used to generate specific configurations of the architectural elements. As surveyed in $\S 2$, ADR models architectures as (hyper)graphs that is a set of (hyper)edges sharing some nodes; respectively, edges represent architectural elements (at some level of abstraction) while nodes represent components' interfaces. Also, ADR production rules take the form $p: L \rightarrow R$ where $L$ is an edge and $R$ an (hyper)graph; rule $p$ is meant to replace $L$ with $R$ within a given graph. The main contribution in [11] is an algorithm that computes a weakest pre-condition $\psi$ out of a post-condition $\varphi$ and a production rule. We prove a theorem that guarantees that the application of the rule to a configuration satisfying $\psi$ yields a configuration satisfying the post-condition $\varphi$. This algorithm can be used to compute a reconfiguration if the current configuration violates the invariant. Roughly, in [11] we envisage architectural styles according to the equation:

$$
\text { architectural style }=\text { production rules }+ \text { invariants }
$$

where an invariant is the property the designer requires of the application.

A summary of our contributions. The main contributions of this paper can be summarised as an extension and a refinement of the methodology in [11].

The extension consists in the adaptation of the methodology to encompass reconfiguration mechanism of ADR. As a matter of fact, ADR features complex reconfigurations that cannot be captured by production rules. Such complex reconfigurations can be envisaged as a model of run-time evolution of systems that describe what complex rearrangements could happen during execution. This is technically done by specifying term rewriting rules in an algebra where terms are interpreted as proofs of the style of graphs. Here we broaden the applicability of the methodology in [11] to a more general setting that allows the iteration of the methodology in [11] when reconfigurations violate the style. A limitation of the methodology in [11] was due to the fact that style violations could be fixed only considering "topdown" application of productions. In this paper we take into account also violations of the style due to complex reconfigurations. To achieve this we have to identify the "positions" in the system where violations occur. This allows us to apply our methodology only to the parts of the system affected by the ill reconfiguration. Also, we propose here a systematic reiteration of the methodology when an immediate way to recover the style cannot be found. This yields a more general and efficient framework.

Intuitively, the equation (1) now becomes

$$
\text { architectural style }=\text { production rules }+ \text { reconfiguration rules }+ \text { invariants }
$$

This generalisation is possible due to the introduction of a monitoring approach that fully exploits the features of ADR.

The refinement we propose here regards the rewriting mechanism of ADR and, more importantly, its underlying monitoring capability. Indeed, as observed in [4], a distinctive aspect of ADR is that it features the canonical view of software architectures in terms of connected architectural elements as well as a hierarchical view of software architectures that is paramount in the design phase. Although [4] advocates 
the use of the architectural view as a useful mechanism to be exploited in complex reconfigurations, no actual formalisation has been provided on how this could be achieved and the parsing features of ADR had been only sketched in [4]. More precisely, we start by proposing minor changes to the original rewriting mechanism of ADR that distinguishes edge as terminal and non-terminal at the type level and allows only non-terminal edges to be rewritten (for each edge type, either all the edges of that type can be rewritten or none of them). Our generalisation eliminates such distinction; an edge can be rewritten if it is marked as "replaceable" in the graph. Therefore, we allow edges of the same type to be rewritten or not depending on how they are marked in the graph. (We note that the original ADR rewriting mechanism can still be obtained: if one decides that an edge type is non-terminal, then all edges of that type have to be replaceable while for types of terminal edges, all edges have to be marked as non-replaceable.) Besides some simplification in the technical presentation of ADR (which is now more uniform), such generalisation brings in extra flexibility. In fact, a replaceable edge can be refined by introducing new versions of a rule that differs only for the "replaceability" of some edges.

In addition we introduce a monitoring mechanism that is also exploited to define an efficient parsing of ADR graphs. Our monitoring mechanism keeps track of the application of reconfiguration rules and uses such information when the graph has to be parsed (to identify the part that violates a style). The application of a reconfiguration rule affects such information that need to be updated accordingly.

Summing up, one can enforce the architectural style of the system in presence of complex reconfiguration that may violate the style; this can be achieved by

1. defining a monitoring mechanism,

2. repeatedly adapting the methodology in [11] exploiting the parsing features defined here.

Structure of the paper. $\S 2$ overviews ADR and introduces its new variant as well as it summarises the results in [11]. $\S 3$ defines our monitoring approach. $\S 4$ gives the new rewriting mechanism hinging on our monitoring approach. $\S 5$ gives the refinement of our methodology. $\S 6$ draws some conclusions.

\section{A Variant of ADR}

In the following, $\mathfrak{N}$ and $\mathfrak{E}$ are two countably infinite and disjoint sets (of nodes and edges respectively), $X^{*} \stackrel{\text { def }}{=}\left\{\left(x_{1}, \ldots, x_{n}\right) \mid x_{1}, \ldots, x_{n} \in X\right\}$ is the set of finite lists on a set $X$, and $\tilde{x}$ ranges over $X^{*}$. Also, abusing notation, we sometimes use $\tilde{x}$ to indicate its underlying set of elements.

Definition 1 ((Hyper)graphs and morphisms [4]) $A$ (hyper)graph is a tuple $G=\langle V, E, t\rangle$ where $V \subseteq \mathfrak{N}$ and $E \subseteq \mathfrak{E}$ are finite and $t: E \rightarrow V^{*}$ is the tentacle function connecting edges $e \in E$ to a list of nodes; the arity of $e$ is the length of $t_{G}(e)$. It is convenient to write $e(\tilde{u}) \in G$ for $e \in E_{G}, t_{G}(e)=\tilde{u} \subseteq V_{G}$; also, given a graph $G, V_{G}, E_{G}$, and $t_{G}$ respectively denote the nodes, the edges, and the tentacle function of $G$.

Given two graphs $G$ and $H$, a morphism from $G$ to $H$ is a pair of functions $\left\langle\sigma_{V}: V_{G} \rightarrow V_{H}, \sigma_{E}: E_{G} \rightarrow\right.$ $\left.E_{H}\right\rangle$ s.t. $\sigma_{V}$ and $\sigma_{E}$ preserve the tentacle functions, i.e. $\sigma_{V}^{*} \circ t_{G}=t_{H} \circ \sigma_{E}$, where $\sigma_{V}^{*}$ is the homomorphic extension of $\sigma_{V}$ to $V_{G}^{*}$.

In ADR, graphs are typed over a fixed type graph via typing morphisms. As usual an ADR graph $G$ is typed over a type graph $\Gamma$ through $\tau_{G}$ if $\tau_{G}$ is a morphism from $G$ to $\Gamma$.

Definition 2 (Typed graphs) Let $\Gamma$ be a type graph. An ADR graph $G$ is a (hyper)graph typed over $\Gamma$ through $\tau_{G}$ if $\tau_{G}$ is a morphism from $G$ to $\Gamma$. 
Example 1 Take the type graph $\Gamma=\langle V, E, t\rangle$ where $V=\{\bullet, \circ\} \subseteq \mathfrak{N}, E=\{C, B, F F, F l s, F l, B F, P, P F\} \subseteq$ $\mathfrak{E}$, and $t: C \mapsto(\bullet), t: B \mapsto(\bullet, \circ)$, and $t: e \mapsto(\bullet, \bullet)$ for each $e \in E \backslash\{B, C\}$.

The graph $G=\left\langle\left\{u_{1}, u_{2}, u_{3}, u_{4}\right\},\left\{f f, f_{1}, f l_{2}\right\}, t^{\prime}\right\rangle$ where $t^{\prime}$ is defined as $t^{\prime}:$ ff $\mapsto\left(u_{2}, u_{1}\right), t^{\prime}: f_{1} \mapsto\left(u_{3}, u_{2}\right)$, and $t^{\prime}: f_{2} \mapsto\left(u_{4}, u_{2}\right)$ can be typed on $\Gamma$ by $\tau_{G}$ mapping all the nodes to $\bullet, f_{1}$ and $f l_{2}$ to $F l s$, and ff to $F F$.

Hereafter, we fix a typed graph $\Gamma$ and tacitly assume that all graphs $G$ are typed over $\Gamma$ via a morphism $\tau_{G}$. Intuitively, $\Gamma$ yields the vocabulary of the architectural elements to be used in the designs; moreover, $\Gamma$ specifies how these elements can be connected together (e.g., as in Example 1).

For technical reasons we introduce a slight variant of ADR; instead of considering edges as nonterminal and terminal edges, the new version of ADR allow more liberal rewriting mechanism by marking in a graph the edges that can be replaced. Technically, this is obtained by considering pairs $\langle G, \theta\rangle$ where $G$ is a graph and $\theta: E_{G} \rightarrow\{0,1\}$ is the replaceability map; an edge $e \in E_{G}$ is replaceable iff $\theta(e)=1$. Abusing notation we will implicitly assume that any graph $G$ is equipped with a replaceablility map which we will denote by $\theta_{G}$.

Type and typed graphs have a convenient visual notation. Nodes are circles and edges are drawn as (labelled) boxes; tentacles are depicted as lines connecting boxes to circles; conventionally, directed tentacles indicate the first node attached to the edge and the others are taken clockwise. The boxes of edges of type graphs are shaded, while the edges in a graph are either single- and double-lined boxes; the former represent non-replaceable edges while the latter represent replaceable ones. The visual notation for typed graphs include the graph and its typing morphism. Nodes are paired with their types while an edge label $e: e^{\prime}$ represents the fact that the typing morphism maps the edge $e$ of the graph to the edge $e^{\prime}$ of the type graph.

Example 2 In the visual notation described above, the type graph $\Gamma$ and the graph $G$ of Example 1 can be respectively drawn as
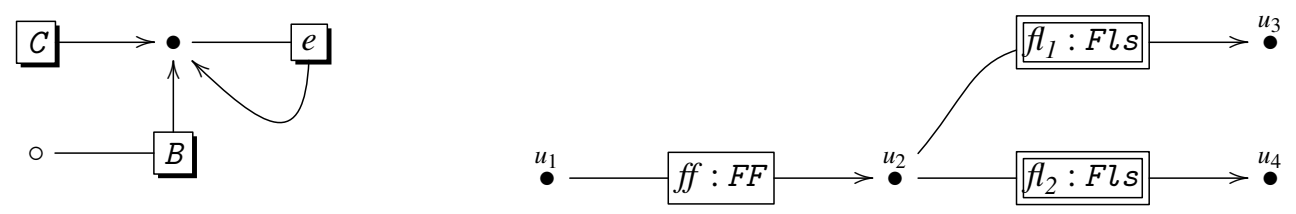

where, to simplify the type graph, we use $e \in E \backslash\{B, C\}$ (instead of drawing an edge for each edge of $\Gamma$ with arity two).

Definition 3 (Typed Graph morphisms) A morphism between $\Gamma$-typed graphs $f: G_{1} \rightarrow G_{2}$ is a typed graph morphism if it preserves the typing, i.e. such that $\tau_{G_{1}}=\tau_{G_{2}} \circ f$.

Note that replaceablility maps are not considered in Definition 2 .

Definition 4 (Productions) $A$ (design) production $p$ is a tuple $\left\langle L, R, i: V_{L} \rightarrow V_{R}\right\rangle$ where $L$ is a graph consisting of a single repleaceble edge attached to distinct nodes and $R$ is a graph; the nodes in Im $(i)$ (the image of $i$ ) are called interface nodes.

Design productions can be thought of as rewriting rules that, when applied to a graph $G$, replace a replaceable (hyper)edge of $G$ matching $L$ with a fresh copy of $R$ (we remark that our morphisms are type-preserving).

Example 3 Take the following graphs:

$$
\begin{aligned}
& G_{L}=\langle\{a, b\},\{f s\}, f s \mapsto(a, b)\rangle \\
& G_{R}=\left\langle\left\{u_{1}, u_{2}, u\right\},\{f l s, p a\}, t_{R}:\left\{\begin{array}{l}
f l s \mapsto\left(u, u_{2}\right) \\
p a \mapsto\left(u_{1}, u\right)
\end{array}\right\rangle\right.
\end{aligned}
$$


with $f s$ of type type Fls, fls of type $F l$, and pa of type $P$. (Note that $G_{L}$ is a single-edge graph.)

The production bookFlight $=\left\langle G_{L}, G_{R}, i_{\text {bookFlight }}\right\rangle$ has $G_{L}$ and $G_{R}$ as left-hand side (LHS) and right-hand side respectively; the interface of bookFlight is given by the map defined as follows:

$$
i_{\text {bookFlight }}:\left\{\begin{array}{l}
a \mapsto u_{1} \\
b \mapsto u_{2}
\end{array}\right.
$$

namely, a (resp. b) corresponds to the first (resp. second) node of $f s$.

Like ADR graphs, productions have an appealing visual representation that we illustrate in the next example that depicts the production of Example 3 .

Example 4 The graphical representation below corresponds to the production bookFlight in Example 3.

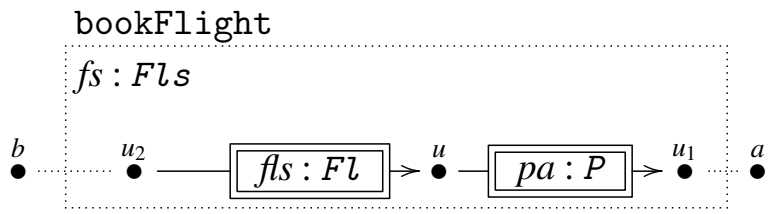

The dotted square and the dotted lines represent the LHS and the map $i_{\mathrm{bookFlight}}$; the name and type of the edge of the LHS is in the top-left corner of the dotted box and the name of the production is given on the top of the dotted square. The RHS of bookFlight is depicted inside the dotted box

The next example illustrates how productions are applied to graphs; the details will be given in $\S 2$ for asserted productions, which encompass ADR productions.

Example 5 Consider the production bookflight of Example 4 Below, the unique edge of type Fls is replaced by an instance of the RHS of bookFlight.

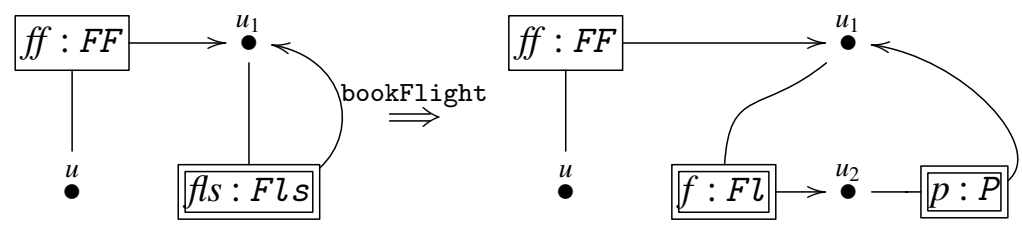

Note that the rest of the graph (consisting only of the edge ff) including the interface nodes is left unchanged while a fresh node $u_{2}$ is created.

We overview the Design by Contract (DbC) approach for ADR introduced in [11]. Note that the variant of ADR given in $\S 2$ generalises the rewriting mechanism originally defined in [4], therefore the results in [11] can be easily adapted to the variant of ADR presented here.

Properties of graphs are expressed in a simple logic tailored for ADR. In the following we let $D, D^{\prime}, \ldots$ range over edges of $\Gamma$.

Definition 5 (ADR logic [11]) Let $\mathrm{V}$ be a countably infinite set of variables for nodes (ranged over by $\mathrm{x}, \mathrm{y}, \mathrm{z}, \ldots)$. The set $\mathscr{L}$ of (graph) formulae for $A D R$ is given by the following grammar:

$$
\psi, \varphi \quad:=\quad \mathrm{x}=\mathrm{y} \quad|\quad \top| \neg \varphi \quad \varphi_{1} \wedge \varphi_{2} \quad \mid \forall D(\tilde{\mathrm{x}}) \cdot \varphi
$$

In formulae of the form $\forall D(\tilde{\mathrm{x}}) \cdot \varphi$, the occurrences of $\mathrm{y} \in \tilde{\mathrm{x}}$ in $\varphi$ are bound, $\tilde{\mathrm{x}}$ has the length of the arity of $D$ and $\tilde{x}$ are pairwise distinct. 
Basically, $\mathscr{L}$ is a propositional logic to predicate on (in)equalities of nodes and it is parametrised with respect to the type graph $\Gamma$ used in quantification. Variables not in the scope of a quantifier are free and the set $\mathrm{fv}(\varphi)$ of free variables of $\varphi \in \mathscr{L}$ is defined accordingly; also, we abbreviate $\mathrm{x}_{1}=\mathrm{x}_{2} \wedge \ldots \wedge \mathrm{x}_{n-1}=\mathrm{x}_{n}$ with $\mathrm{x}_{1}=\mathrm{x}_{2}=\ldots=\mathrm{x}_{n-1}=\mathrm{x}_{n}$ and we define $\perp$ as $\neg \mathrm{T}, \mathrm{x} \neq \mathrm{y}$ as $\neg(\mathrm{x}=\mathrm{y}), \varphi \vee \psi$ as $\neg(\neg \varphi \wedge \neg \psi)$, $\varphi \rightarrow \psi$ as $\neg \varphi \vee \psi$, and $\exists D(\tilde{x}) . \varphi$ as $\neg \forall D(\tilde{x}) . \neg \varphi$. The models of $\mathscr{L}$ are ADR graphs together with an interpretation of the free variables of formulae.

Definition 6 (Satisfaction relation) A graph $G$ satisfies $\varphi \in \mathscr{L}$ under the assignment $h: \mathrm{V} \rightarrow V_{G}$ (in symbols $\left.G=_{h} \varphi\right)$ iff

$$
\begin{aligned}
& \varphi \equiv \top, \quad \text { or } \\
& \varphi \equiv \mathrm{x}=\mathrm{y} \quad \text { and } \quad h(\mathrm{x})=h(\mathrm{y}), \quad \text { or } \\
& \varphi \equiv \neg \varphi^{\prime} \quad \text { and } G \not \nvdash_{h} \varphi^{\prime} \text {, or } \\
& \varphi \equiv \varphi_{1} \wedge \varphi_{2} \quad \text { and } \quad G=_{h} \varphi_{1} \text { and } G \models_{h} \varphi_{2}, \quad \text { or } \\
& \varphi \equiv \forall D(\tilde{\mathrm{x}}) . \varphi \quad \text { and }\left.\quad G\right|_{h[\tilde{\mathrm{x}} \mapsto \tilde{u}]} \varphi \text { for any } d(\tilde{u}) \in G \quad \text { s.t. } \tau_{G}(d)=D
\end{aligned}
$$

Note that in the last clause of Definition 6, each bound variable in $\tilde{x}$ is instantiated with a node. It is easy to prove that we can restrict to finite mappings that only assign the free variables of formulae. Namely, for each $h, h^{\prime}: \mathrm{V} \rightarrow V_{G}$, if $\left.h\right|_{\mathrm{fv}(\varphi)}=\left.h^{\prime}\right|_{\mathrm{fv}(\varphi)}$ then $\left.G\right|_{h} \varphi$ iff $G=_{h^{\prime}} \varphi$. We write $G \models \varphi$ when $\mathrm{fv}(\varphi)=\emptyset$.

Example 6 Consider the formulae

$$
\begin{array}{rll}
\text { noEdge }\langle D\rangle & \stackrel{\text { def }}{=} \forall D(\tilde{\mathrm{x}}) \cdot \perp \\
\phi_{e x} & \stackrel{\text { def }}{=} \quad \forall D(\mathrm{x}, \mathrm{y}) \cdot \exists D^{\prime}(\mathrm{z}) \cdot \mathrm{x}=\mathrm{z}
\end{array}
$$

Formula (2) characterises the graphs that do not contain edges of a given type while the formula (3) describes graphs such that each edge of type $D$ is connected to one of type $D^{\prime}$ on the first tentacle. For instance, consider the graphs

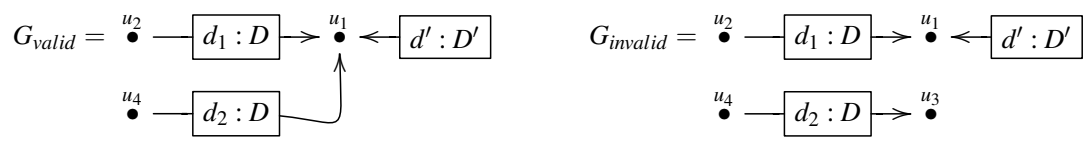

then $G_{v a l i d}$ satisfies $\phi_{e x}$ whereas $G_{\text {invalid }}$ does not, because $d_{2}$ is not connected to any edge of type $D^{\prime}$.

Fix an ADR production $p=\langle L, R, i\rangle$. Our notion of contracts hinges on asserted productions, namely ADR productions decorated with pre- and post-conditions expressed in the logic $\mathscr{L}$. Given $\psi, \varphi \in \mathscr{L}$ and two assignments $h, h^{\prime}: \mathrm{V} \rightarrow \mathfrak{N}$, an asserted production is an expression of the form

$$
\{\psi, h\} p\left\{\varphi, h^{\prime}\right\} \quad \text { where } \quad h(\mathrm{fv}(\psi)) \subseteq V_{L} \text { and } h^{\prime}(\mathrm{fv}(\varphi)) \subseteq V_{R}
$$

An asserted production generalises ADR productions and it intuitively requires that if $p$ is applied to a graph $G$ that satisfies $\psi$ then the resulting graph is expected to satisfy $\varphi$. The assignments $h$ and $h^{\prime}$ in (4) allow pre- and post-conditions to predicate on nodes occurring in the LHS or the RHS of $p$.

Operationally, an asserted production $\pi$ can be applied to a graph $G$ by replacing an "instance" of the LHS in $G$ (identified by an matching homomorphism) with a new instance of the RHS and connecting the interface nodes accordingly, provided that $G$ satisfies the precondition of $\pi$ (under the matching homomorphism). For the variant of ADR proposed in $\S 2$ we just have to impose the condition that the homomorphic image of the LHS of $\pi$ has to be a replaceable edge. This is schematically illustrated in Figure 1 (cf. [11]) and demonstrated in Examples 7 and 8 . 


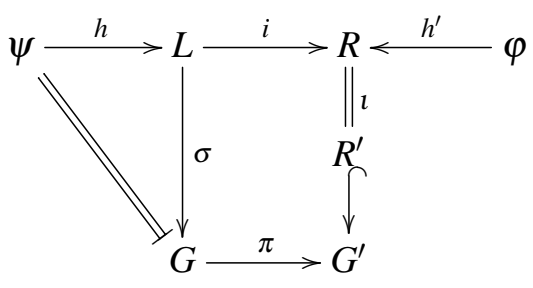

Figure 1: Asserted design productions

Example 7 Consider the production bookFlight given in Example 4 and the asserted production

$$
\pi \stackrel{\text { def }}{=}\{\psi, \emptyset\} \text { bookFlight }\{\top, \emptyset\} \quad \text { where } \quad \psi \stackrel{\text { def }}{=} \forall F l s(\mathrm{x}, \mathrm{y}) . \mathrm{x} \neq \mathrm{y}
$$

Then, $\pi$ cannot be applied to the leftmost graph $G$ in the rewriting of Example 5 because $G \not \models \psi$ (under the unique morphism $\sigma$ from $L$ to $G$ ). In fact, $\mathrm{x}$ and $\mathrm{y}$ are mapped to the same node $u_{1}$ of $G$.

Example 8 The rewriting below is obtained by applying $\pi$ in Example 7

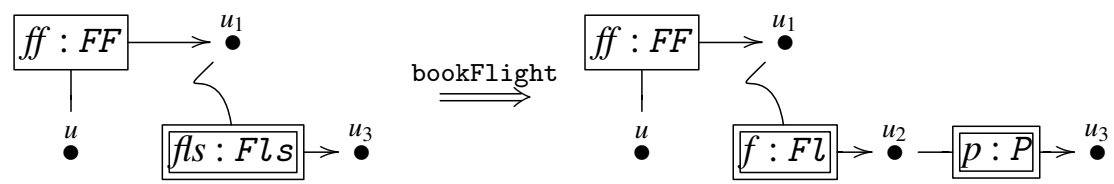

The edge fls is replaced by an isomorphic instance of $R$ preserving the interface nodes $u_{1}$ and $u_{3}$.

Note that the application of an asserted production generalises the hyper-edge replacement mechanism of ADR; in fact, $\{\top, \emptyset\} p\{\top, \emptyset\}$ applies exactly as normal ADR productions.

An asserted production $\pi$ is valid when any application of $\pi$ to a graph satisfying the precondition of $\pi$ yields a graph satisfying the post condition of $\pi$. Obviously, not all asserted productions $\{\psi, h\} p\left\{\varphi, h^{\prime}\right\}$ are valid (this can be trivially noted by taking $\varphi$ to be $\perp$ ). In [11] we define an algorithm $1 \mathscr{W}_{h^{\prime}}(p, \varphi)$ that, given a production $p$ and a post-condition $\varphi$, returns a weakest pre-condition $\psi$ so that $\{\psi, h\} p\left\{\varphi, h^{\prime}\right\}$ is valid.

The next example is adapted from [11].

Example 9 Consider $\varphi \in \mathscr{L}$ and the production pay below:

$$
\varphi \stackrel{\text { def }}{=} \forall B(\mathrm{x}, \mathrm{y}) \cdot \forall C(\mathrm{z}) \cdot \mathrm{y}=\mathrm{z} \quad \text { pay } \stackrel{\text { def }}{=} \quad \begin{aligned}
& P \\
& \stackrel{\circ}{=}-b: B>\bullet
\end{aligned}
$$

We remark that the post-condition $\varphi$ requires that every edge of type $B$ is connected (on its second tentacle) to every edge of type $C$. The computed weakest pre-condition is

$$
\mathscr{W}_{\emptyset}(\text { pay }, \varphi)=\text { noEdge }\langle C\rangle \wedge \forall B(\mathrm{x}, \mathrm{y}) \cdot \forall C(\mathrm{z}) \cdot \text { noEdge }\langle C\rangle \wedge \forall B(\mathrm{x}, \mathrm{y}) \cdot \forall C(\mathrm{z}) \cdot \mathrm{y}=\mathrm{z}
$$

We remark that $\mathscr{W}_{\emptyset}($ pay, $\varphi)$ imposes that for the validity of the asserted production it is necessary that the graph does not have any edges of type $C$. In fact, production pay will generate an edge of type $B$ whose second tentacle is attached to an internal node $u$ that cannot be shared with any edge of type $C$ already appearing in the graph.

\footnotetext{
${ }^{1}$ For simplicity, we ignore the assignments and environments that the algorithm in [11] uses to compute weakest preconditions.
} 


\section{Tracking ADR Architectural Reconfigurations}

A key aspect of ADR is to envisage systems as ensembles of designs, that is components with interfaces. Designs are supposed to be generated by means of productions and can be subject to run-time reconfigurations modelled as reconfiguration rules. The use of productions yields two pivotal ingredients of ADR. For clarity, we consider only productions as it is just a matter of technicality to adapt this section to asserted productions.

Firstly, productions implicitly equip designs with a hierarchical structure that can be formalised as the "derivation tree" determining the design. In fact, a set of ADR productions induces a multi-sorted algebraic signature $\Sigma$ where the sorts are the type edges in the type graph ${ }^{2}$ and the operations are the productions themselves, once a total order on the edges in the RHS of the production is fixed. Hereafter, we fix such an order ${ }^{3}$ and, given the RHS $R$ of a production, we write $R[j]$ for the $j$-th edge in $R$. With this construction, an ADR production becomes an operation with type

$$
E_{1} \times \ldots \times E_{n} \rightarrow L
$$

where $E_{k}$ is the type of the $k$-th edge in the RHS of the production (according to the chosen order on edges in the RHS) and $L$ is the type of the edge in the LHS. In other words, an ADR production like in (5) can be envisaged as an operation in some algebras of designs that builds a design $G$ of type $L$ out of designs $G_{k}$ of type $E_{k}$ (for $1 \leq k \leq n$ ). This corresponds to a "bottom-up" development (whereby designs are assembled out of other components) and, as observed in [4], it parallels the "top-down" generation of designs (similar to context-free grammars) reviewed in $\S 2$. Moreover, one could consider the terms (with sorted variables to model partial designs) built on $\Sigma$ and adopt the obvious operational interpretation: $G$ is obtained by replacing the $j$-th edge in the RHS with $G_{j}$ (and connecting the interface nodes as appropriate). The elements of such term algebra correspond to the proof that a given design can be assigned some type.

Example 10 The production bookFlight in Example 4 yields the operation

$$
\text { bookFlight : } F l \times P \rightarrow F l s
$$

assuming that in the chosen order, $f$ is smaller than $p$.

Secondly, ADR exploits the algebraic view of productions to model complex architectural reconfigurations that cannot be captured by productions. In fact, design can evolve for instance when components have to be removed, added, or assembled in a different way. Architectural reconfigurations are naturally modelled as transformation of elements in the $\Sigma$-term algebra with variables $\operatorname{Term}_{\Sigma, \mathscr{X}}$ (where $\mathscr{X}$ is the set of variables). Formally, this is achieved by defining a term rewriting system on $\operatorname{Term}_{\Sigma, \mathscr{X}}$; namely, a reconfiguration rule takes the form

$$
t \rightarrow t^{\prime}
$$

where $t, t^{\prime} \in \operatorname{Term}_{\Sigma, \mathscr{X}}$ are linear terms (that is each variable occurs at most once in $t$ and similarly for $t^{\prime}$ ) and the variables occurring in $t^{\prime}$ also occur in $t$.

Example 11 Combining the operation in Example 10 with the one associated to the production in Example 4 one could build the term bookFlight $(x, \operatorname{pay}(y))$ of type $F l s$ (provided that $x$ is of type $F l$ and $y$ is of type $B$.

\footnotetext{
${ }^{2}$ In the original ADR presentation, the sorts are just the non-terminal edges. In our variant, this can be simplified by taking all edges of the type graph as sorts.

${ }^{3}$ The chosen order is completely arbitrary and does not affect the construction described above.
} 
Below we give an example of simple reconfigurations.

Example 12 Consider the following productions:
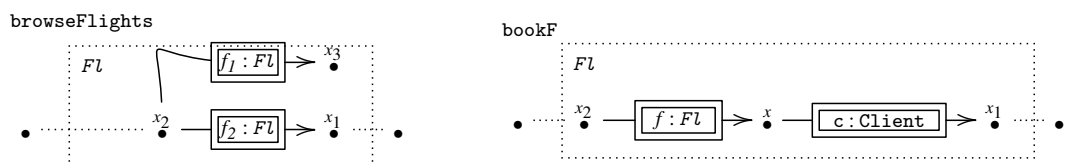

We can define the following reconfiguration rule:

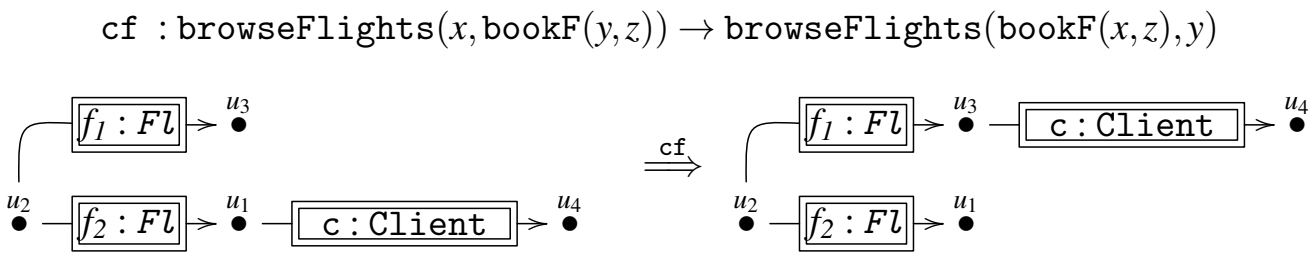

Observe that, unlike in the application of ADR productions, the identity of edge $c$ is preserved when applying the reconfiguration rule $c f$. Also, for simplicity in Example12 we take $c$ to be just a single edge, but the effect of $\mathrm{cf}$ would be the same if instead of edge $c$ we had a complex graph of type Client: the whole graph would have been moved from node $u_{1}$ to node $u_{4}$.

A result in [4] shows that the simple condition that an ADR rewriting system where all reconfiguration rules of form (6) have $t$ and $t^{\prime}$ of the same sort guarantees that the architectural style is preserved when the system evolves.

In this paper we exploit the algebraic presentation of ADR production and reconfiguration mechanisms and combine them together with a tracking mechanism that is used to recover possible run-time misbehaviour.

Definition 7 below formalises our tracking mechanism using some trees to record graphs' evolution due to productions and reconfigurations respectively. We introduce some technical machinery first.

We consider forests of trees with vertexes drawn from a set $\mathrm{N}$ (hereafter we will call the nodes of the trees vertices's in order to distinguish them from the graph's nodes); if $f: X \rightarrow Y$ is a partial map then we write $f(x) \uparrow$ when $f$ is undefined on $x$ and we let dom $f=X \backslash\{x \in X \mid f(x) \uparrow\}$. Hereafter, we fix a finite set of productions $\mathscr{P}$ to denote all the productions of the system. A tracking Environment $\mathscr{T}$ is pair of two injective finite partial maps

$$
\mathscr{T}^{(1)}: \mathrm{N} \rightarrow \mathfrak{E} \times \mathfrak{N}^{*}, \quad \text { and } \quad \mathscr{T}^{(2)}: \mathrm{N} \rightarrow \mathscr{P},
$$

and we use $\mathbf{0}$ to denote the empty environment (that is the environment undefined on all $\mathrm{n} \in \mathrm{N}$ ).

Basically, given a forest $T$, we use an environment $\mathscr{T}$ (such that dom $\mathscr{T}$ is the set of vertices's of $T$ ) so to decorate each vertex of $T$ with two attributes:

- $\mathscr{T}^{(1)}(\mathrm{n})$ assigns an edge with its list of nodes to the vertices's of $T$, and

- $\mathscr{T}^{(2)}(\mathrm{n})$ assigns a production to the vertex $\mathrm{n}$ in $T$.

It is convenient to write $\mathscr{T}(\mathrm{n}) \stackrel{a s}{=} e(\tilde{x}) \cdot p$ when $\mathscr{T}^{(1)}(\mathrm{n})=e(\tilde{x})$ and $\mathscr{T}^{(2)}(\mathrm{n})=p$. Also, in the following we use a notation inspired by object-oriented programming to manipulate trees; more precisely, we consider trees $T$ (and their nodes $\mathrm{n}$ ) as objects and write $T$.addTree $\left(\mathrm{n}, T_{1}, \ldots, T_{k}\right)$ to add the trees $T_{h}$ as sub-trees of $T$ by rooting them at the vertex $\mathrm{n}$ in $T$; that is, the resulting tree will be $T$ where vertex $\mathrm{n}$ has the root of $T_{1}, \ldots, T_{k}$ as new children. Also, we let deg $\mathrm{n}$ to be the degree of a vertex $\mathrm{n}, \mathrm{n}[j]$ to be its $j$-th child, and (abusing notation) we allow ourselves to identify trees consisting only of a root with the root vertex. 
Definition 7 (Tracking productions) Let $G_{0}, \ldots, G_{m}$ be a sequence of graphs s.t. for each $0 \leq j<m$, $G_{j+1}$ is obtained from $G_{j}$ by applying a production $p_{j} \in \mathscr{P}$ with morphisms $\sigma_{j}^{\prime}: L_{j} \rightarrow G_{j}$ and $\sigma_{j}: R_{j} \rightarrow$ $G_{j+1}$ where $L_{j}$ and $R_{j}$ are the LHS and RHS of $p_{j}$, respectively.

We associate to each $G_{j}$ a tracking forest $T_{j}$ and a tracking environment $\mathscr{T}_{j}$ as follows:

- let $r$ be the number of edges in $G_{0}$, forest $T_{0}=\mathrm{n}_{1}, \ldots, \mathrm{n}_{r}$ consists of $r$ single-vertex trees with roots $\mathrm{n}_{1}, \ldots, \mathrm{n}_{r}$ taken pairwise distinct. Environment $\mathscr{T}_{0}$ is defined as the map that takes the $m$-th vertex in the forest $T_{0}$ to the $m$-th edge of $G_{0}$; formally,

$$
\mathscr{T}_{0}\left[\mathrm{n}_{m} \mapsto e_{m}\left(\tilde{x}_{m}\right) \cdot \uparrow\right] \quad \text { for } \quad 1 \leq m \leq r
$$

where $e_{m}=G_{0}[m]$ and $\tilde{x}_{m}$ are the nodes in $G_{0}$ that $e_{m}$ is attached to;

- Let $k_{j}$ be the number of edges in the RHS of $p_{j}$ (that is, $k_{j}$ is the cardinality of $E_{R_{j}}$ ), and let $\mathrm{n}$ be the inverse image of $\sigma_{j}^{\prime}\left(e_{j}\left(\tilde{x}_{j}\right)\right)$ through $\mathscr{T}_{j}^{(1)}$ s.t. $\mathscr{T}_{j}^{(1)}(\mathrm{n})=\sigma_{j}^{\prime}\left(e_{j}\left(\tilde{x}_{j}\right)\right)$ and, for $1 \leq l \leq k_{j}$, let $T_{l}^{\prime}$ be a tree made of just a fresh vertex node, then

$$
T_{j+1}=T_{j} \text {.addTree }\left(\mathrm{n}, T_{1}^{\prime}, \ldots, T_{k_{j}}^{\prime}\right)
$$

Environment $\mathscr{T}_{j+1}$ is obtained by updating $\mathscr{T}_{j}$ in the following way:

$$
\mathscr{T}_{j+1}= \begin{cases}\mathscr{T}_{j}\left[\mathrm{n} \mapsto \sigma_{j}^{\prime}\left(e_{j}\left(\tilde{x}_{j}\right)\right) \cdot p_{j}, T_{l}^{\prime} \mapsto \sigma_{j}\left(R_{j}[l]\right) \cdot \uparrow \mid l=1, \ldots, k\right] & \text { if } E_{R_{j}} \neq \emptyset \\ \mathscr{T}_{j}\left[\mathrm{n} \mapsto \sigma_{j}^{\prime}\left(e_{j}\left(\tilde{x}_{j}\right)\right) \cdot p_{j}, T_{1}^{\prime} \mapsto \uparrow \cdot \uparrow\right] & \text { if } E_{R_{j}}=\emptyset\end{cases}
$$

Despite some technical intricacy, Definition 7 is conceptually simple. Basically, we add to $T_{j}$ as many fresh vertexes as the edges in the RHS of the production $p_{j}$; such vertexes become the children of the vertex $\mathrm{n}$ in $T_{j}$ associated with the LHS $\sigma_{j}^{\prime}\left(e_{j}\right)$. Accordingly, the environment $\mathscr{T}_{j+1}$ updates $\mathscr{T}_{j}$ recording edges and productions associated to $\mathrm{n}$ and the fresh roots of $T_{l}^{\prime}$. Observe that each forest $T_{j}$ has $r$ trees, with $r$ the number of edges of $G_{0}$. Indeed, the evolution of $G_{0}$ involves only the replacement of such edges (and those produced by such replacements). Therefore, we can record the application of $p_{j}$ to $G_{j}$ in a node of one of the trees representing the evolution of one of the initial edges of $G_{0}$.

Example 13 Consider the production browseFlights from Example 12 For presentation purposes we use $p_{j}(e)$ to indicate the $j$-th application of the production browseFlights on an edge e.

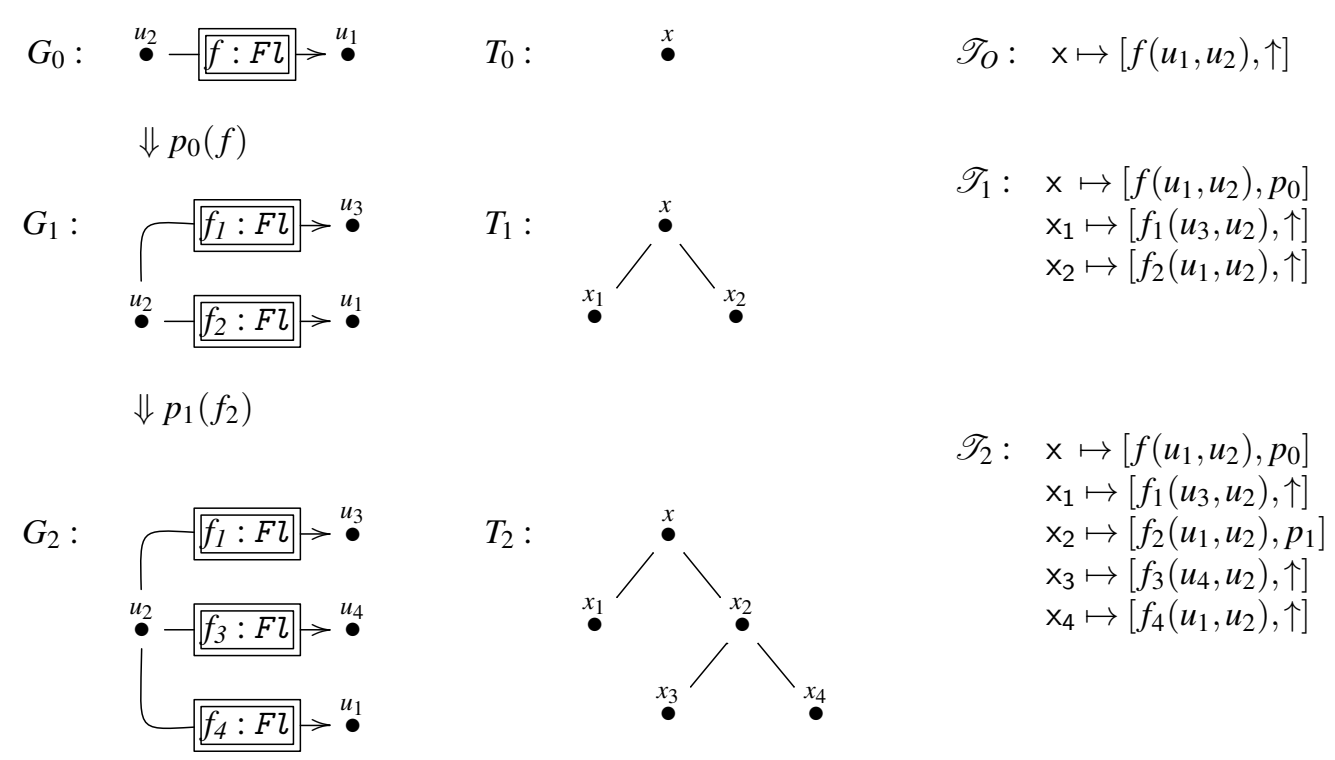


Graph $G_{0}$ represents the initial graph, $T_{0}$ its corresponding tree mapped to an initial environment $\mathscr{T}_{0}$ as defined in Definition 7 By applying browseFlights to $f$ we obtain $G_{1}$. Since the RHS of browseFlights generates two edges we have to add two nodes in $T_{0}$ as children of the node corresponding to $f$ to obtain $T_{1}$. Finally, we update the map of $\times$ in $\mathscr{T}_{0}$ to add the production applied to $f$ and add two new mappings for the fresh nodes of $T_{1}$ to get $\mathscr{T}_{1}$. We now repeat this procedure for every application of a production.

Hereafter, we will show the environment $\mathscr{T}$ within the tree $T$ as shown in the example below.

Example 14 This example shows a simplified way of representing the tree $T$ together with the environment $\mathscr{T}$. We borrow the tree $T_{1}$ and the environment $\mathscr{T}_{1}$ from Example 13 to show this visual simplification.

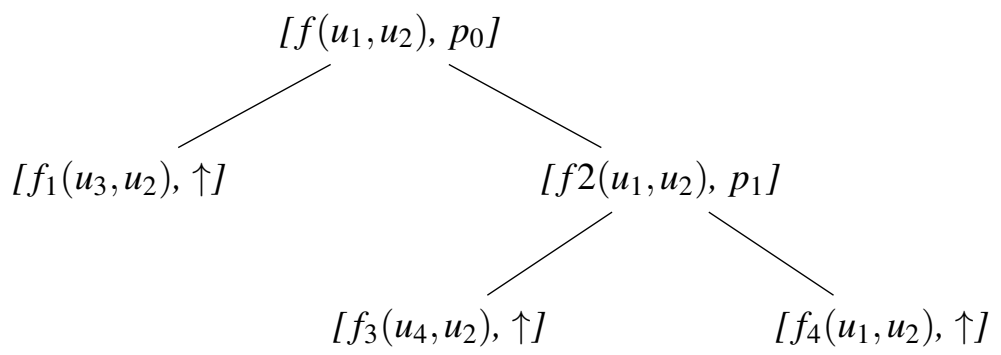

\section{A new rewriting mechanism}

Following the recording mechanism in Definition 7 we define a new rewriting mechanism for ADR reconfigurations. Our approach hinges on tracking trees similar to those in Definition 7 . The new rewriting approach will also allow us to keep track of changes due to reconfigurations.

Given a term $t \in \operatorname{Term}_{\Sigma, \mathscr{X}}$ and a tracking tree $T$, the bow tie relation $t \bowtie T$ holds iff $t$ and $T$ are isomorphic "up to the leaves" of $t$; more precisely, the tree obtained by considering just the internal nodes of $t$ is isomorphic to $T$. This is formalised in Definition 8

Definition 8 (Bow tie relation) The relation $\bowtie$ in defined as

$$
t \bowtie T \quad \Longleftrightarrow \quad t \in \mathscr{X} \quad \text { or } \quad \mathscr{T}^{2}(\diamond)=p \wedge t=p\left(t_{1}, \ldots, t_{k}\right) \wedge \bigwedge_{j=1, \ldots, k} t_{j} \bowtie \diamond[j]
$$

where $\diamond$ is the root of $T$.

Given a reconfiguration rule $\rho: t \rightarrow t^{\prime}$, relation $\bowtie_{-}$given in Definition 8 allows us to identify which parts of a graph match the LHS of $\rho$ exploiting the correspondence between tracking trees and graphs. A sub-tree $T^{\prime}$ of $T$ matches $t$ iff $t \bowtie T^{\prime}$. Assuming $t \bowtie T^{\prime}$ holds then given a variable $x$ occurring in $t$, Definition 9 returns the sub-tree of $T^{\prime}$ (say $T_{x}$ ) corresponding to $x$. This is obtained by applying $t \sqsubset_{x} T^{\prime}$ and is formalised below.

Definition 9 (Tree of a variable) Let $x$ be a variable occurring in $t, \diamond$ be the root of $T, 1 \leq j \leq k$, and $x \in t_{j}$ denote that $t_{j}$ contains variable $x$ then

$$
t \sqsubset_{x} T= \begin{cases}T & \text { if } t=x \\ t_{j} \sqsubset_{x} \diamond[j] & \text { if } \mathscr{T}^{(2)}(\diamond)=p \wedge t=p\left(t_{1}, \ldots, t_{k}\right) \wedge x \in t_{j}\end{cases}
$$

returns the subtree of $T$ corresponding to $x$ if $T$ can mach a path from the root of $t$ to $x$. 


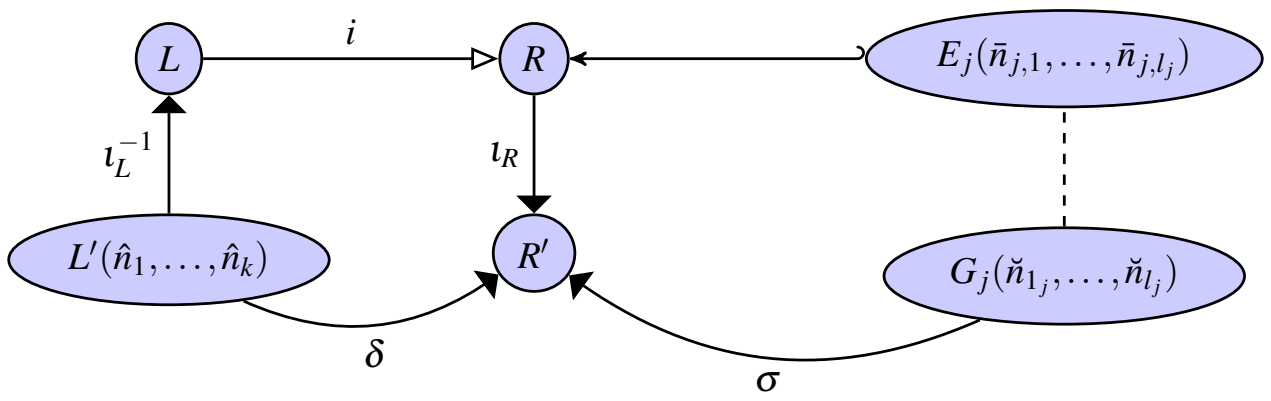

Figure 2: Term to graph morphisms and maps (cf. Definition 10)

Definition 10 builds a graph $\gamma(t)$ out of a term $t \in \operatorname{Term}_{\Sigma, \mathscr{X}} \cdot \gamma$ inspects $t$ inductively and generates a graph corresponding to the productions associated to $t$. In the places of the variables of $t, \gamma$ generates an edge of the appropriate type. More precisely $\gamma(t)$ returns a triplet of fresh edges, nodes, and a mappingfunction that relates variables of $t$ the fresh edges generated. For clarity, in Fig. 2 we provide a visual view of the morphism and mappings of Definition 10.

Definition 10 (From Term to Graph) Let $p=\left\langle L, R, i: V_{L} \rightarrow V_{R}\right\rangle \in \mathscr{P}$ and $t \in \operatorname{Term}_{\Sigma, \mathscr{X}}$.

$$
\gamma(t)= \begin{cases}\left(e,\left[n_{1}, \ldots, n_{h}\right], \eta: t \mapsto e\right) & \text { if } t \in \mathscr{X} \\ \left(\left(G_{1} \cup \cdots \cup G_{r}\right) \sigma, \delta, \eta_{1} ; \sigma \cup \cdots \cup \eta_{r} ; \sigma\right) & \text { if } t=p\left(t_{1}, \ldots, t_{r}\right), \\ & \gamma\left(t_{j}\right)=\left(G_{j}, \delta_{j}, \eta_{j}\right) \text { for } 1 \leq j \leq r\end{cases}
$$

where in the first clause,

- $e$ is a fresh edge of the type corresponding to $t$,

- $\left[n_{1}, \ldots, n_{h}\right]$ are its (e's) fresh pairwise distinct nodes and

- $\eta$ is the mapping of the variable $t$ to the fresh edge $e$

and in the second clause,

- $\delta_{j}=\left[\breve{n}_{1_{j}}, \ldots, \breve{n}_{l_{j}}\right]$ for each $1 \leq j \leq r$ and

- if $\imath_{L}: L \rightarrow L^{\prime}$ and $\imath_{R}: R \rightarrow R^{\prime}$ are the isomorphisms from $L$ and $R$ to two fresh graphs $L^{\prime}$ and $R^{\prime}$ (s.t. all their nodes and edges are fresh) respectively then

- $\delta=\imath_{R}\left(i\left(\imath_{L}^{-1}\left(\left[\hat{n}_{1}, \ldots, \hat{n}_{k}\right]\right)\right)\right)$ where $\left[\hat{n}_{1}, \ldots, \hat{n}_{k}\right]$ are the nodes of $L^{\prime}$ and

- for $1 \leq m \leq l_{j}, \quad \sigma: \breve{n}_{m} \mapsto \imath_{R}\left(\bar{n}_{j, m}\right)$ where $\left[\bar{n}_{j, 1}, \ldots, \bar{n}_{j, l_{j}}\right]$ are the nodes of the $j$-th edge of $R$.

Definition 10 builds a graph $\gamma(t)$ out of a term $t$. Intuitively, $\gamma$ inspects $t$ "bottom-up" and it associates disjoint designs (graphs $G_{j}$ with interfaces $\delta_{j}$ ) to each sub-term of $t$ (note that fresh edges attached to fresh nodes are associated to each variable of $t$ ); then $\gamma$ composes the disjoint designs according to the production $p$ which is rendered by replacing the nodes through the substitution $\sigma$ in Definition 10 .

Definition 11 below establishes how to apply a reconfiguration rule to a graph.

Definition 11 (Applying reconfiguration rules) Fix a reconfiguration rule $\rho: t \rightarrow t^{\prime}$ with $X$ being the set of variables of $t$, a graph $G$, and a tracking tree $T$ of $G$ with the corresponding environment $\mathscr{T}$; let $T^{\prime}$ be a sub-tree of $T$ such that $t \bowtie T^{\prime}$. For $x \in X$, let $T_{x}^{\prime}=t \sqsubset_{x} T^{\prime}$ be the sub-tree of $T^{\prime}$ corresponding to $x$. An application of $\rho$ to $G$ wrt $T$ is a graph $G^{\prime}=G\left[G_{L} \mapsto G_{t}\right]$ where 
- $G_{L}=\bigcup_{l=1}^{m} \mathscr{T}^{(1)}\left(\mathrm{n}_{l}\right)$ where $\mathrm{n}_{1}, \ldots, \mathrm{n}_{m}$ are the leaves of $T^{\prime}$, and

- $G_{t}=\gamma\left(t^{\prime}\right)\left[\eta(x) \mapsto G_{x} \mid x \in X\right]$ where $G_{x}$ is the sub-graph of $G$ corresponding to $x$ and it is defined as $G_{x}=\bigcup_{l=1}^{h_{j}} \mathscr{T}^{(1)}\left(\mathrm{n}_{l}\right)$ with $\mathrm{n}_{j, 1}, \ldots, \mathrm{n}_{j, h_{j}}$ being the leaves of $T_{x}^{\prime}$.

Finally, given the sub-trees $T_{x}^{\prime}$ computed above, we replace the tree $T^{\prime}$ in $T$ with a fresh sub-tree $T^{\prime \prime}$ corresponding to $t^{\prime}$ where we replace the vertexes corresponding to the variables of $t^{\prime}$ with the appropriate sub-trees $T_{x}^{\prime}$. We then update the environment $\mathscr{T}$ so that it maps all the nodes of $T^{\prime \prime}$ up to the sub-trees $T_{x}^{\prime}$ to the productions associated to them through $t^{\prime}$.

We observe that, using Definition 10. Definition 11 simply replaces the graph corresponding to $\gamma(t)$ with the graph corresponding to $\gamma\left(t^{\prime}\right)$ where the edges corresponding to the variables of $t^{\prime}$ are replaced by the corresponding sub-graphs of $G$ identified through the proper morphisms.

Example 15 Let us consider again the productions cf in Example 12; for readability here we use t and $t^{\prime}$ to refer to the LHS and RHS of cf respectively and we also abbreviate browseFlights with brF:
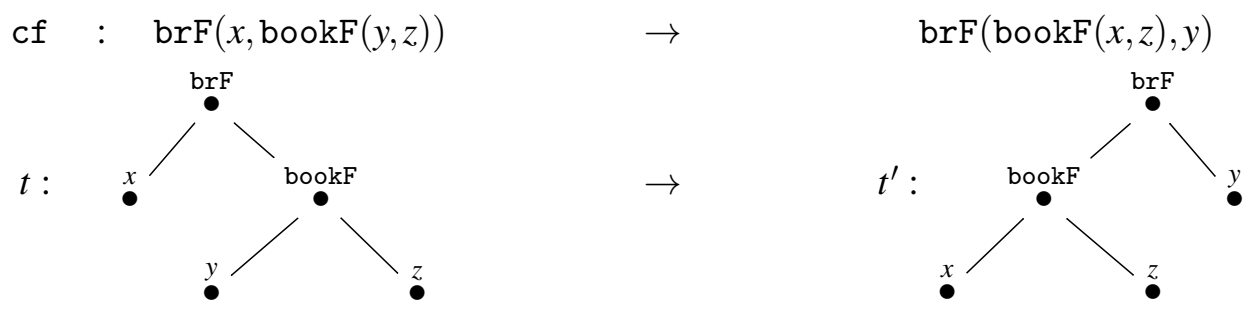

Fix an environment $\mathscr{T}$ and consider the graph $G$ below:

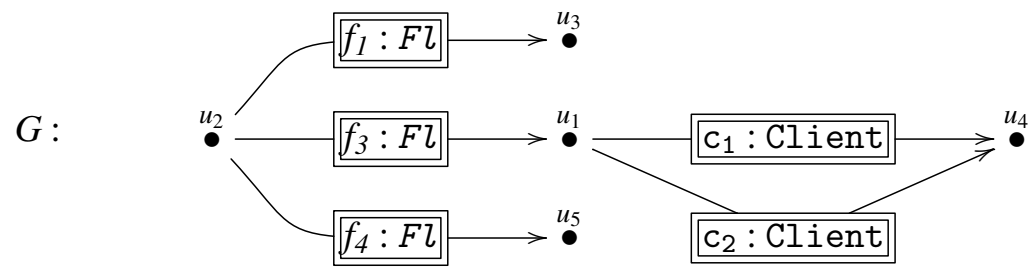

To apply cf to $G$ we have to identify a tree in the forest tracking $G$; this is done inspecting each tree of

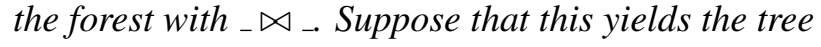

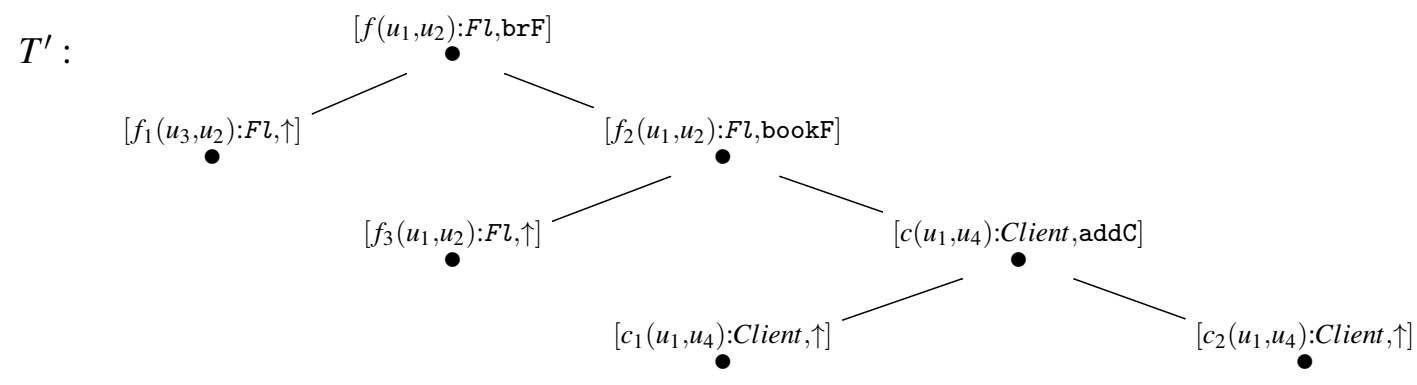

such that $t \bowtie T^{\prime}$, then 
1. using $t \sqsubset T^{\prime}$ we can obtain the sub-tree of $T^{\prime}$ corresponding to each variable of t and get

$$
\begin{aligned}
& t^{x} \sqsubset_{x} T^{\prime}={ }^{\left[f_{1}\left(u_{3}, u_{2}\right): F l, \uparrow\right]} \bullet \quad t^{y} \sqsubset_{y} T^{\prime}={ }^{\left[f_{3}\left(u_{1}, u_{2}\right): F l, \uparrow\right]}
\end{aligned}
$$

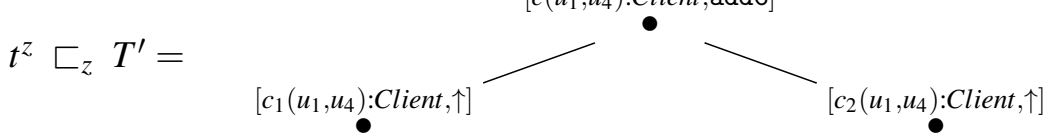

By taking the union of all the edges mapped through $\mathscr{T}$ to the leaves to the sub-trees corresponding to each variable we obtain sub-graphs of $G$ corresponding to each one. Observe that $z$ is not mapped to a single node $T^{\prime}$. This is the reason we need to take the union of all the edges corresponding to the leaves of $t^{z} \sqsubset_{z} T^{\prime}$ in order to obtain its sub-graph.

2. identify the sub-graph of $G$ corresponding to $T^{\prime}$; similarly to step 1 we take the union of all the edges mapped through $\mathscr{T}$ to the leaves of $T^{\prime}$ to obtain:

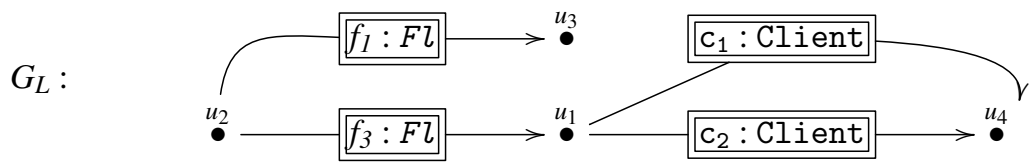

3. use $\gamma\left(t^{\prime}\right)$ to construct a graph corresponding to $t^{\prime}$

$$
\gamma\left(t^{\prime}\right):
$$

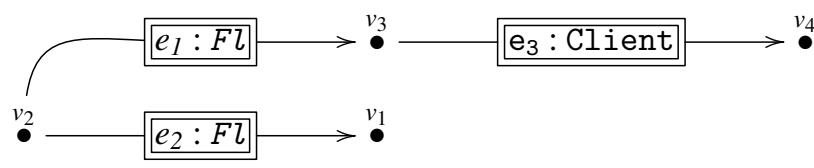

4. $\gamma\left(t^{\prime}\right)$ represents the graph corresponding to $t^{\prime}$ where in the place of the variables it contains a dummy edge of the appropriate type. This is where step 1 comes in place. We replace all the dummy edges in $\gamma\left(t^{\prime}\right)$ with the graphs corresponding to the variables of the dummy edges to obtain $G_{t^{\prime}}$.

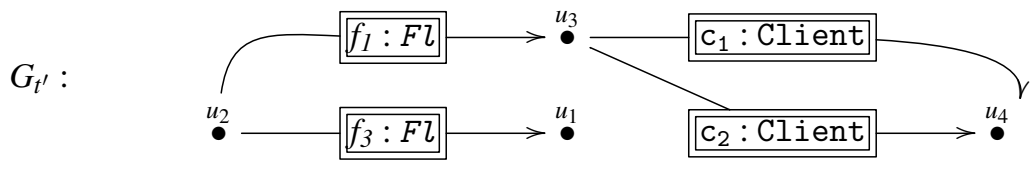

5. the last step requires that we replace $G_{L}$ in $G$ with $G_{t^{\prime}}$.

\section{Recovering invalid configurations}

In [11] we gave a basic methodology for recovering a system to a valid state when a run-time configuration compromises its architectural style. The main objective of our approach in [11] can be observed in Example 16 below.

Example 16 ( [11]) Consider the run-time reconfiguration

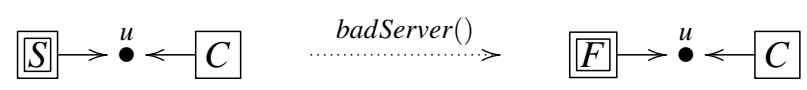

where $S$ changes as illustrated to model a failure $F$. By imposing an invariant that states that every client has to be connected to a non-failed server, the invalid configuration can be identified and recovered. 
The fact that we can now record the evolution of our graphs gives us many advantages. The monitoring mechanism introduced in 3 and the way we apply reconfigurations now gives us the potential to identify which part of the graph (say $G$ ) has been re-written. Using the tree corresponding to the affected sub-graph (say $G_{R}$ ) one can identify the last production applied by observing the parent node of the leaves corresponding to $G_{R}$.

By using the monitoring system one can now spot where a violation may have occurred and attempt to fix such violations without considering or parsing the whole graph in every possible way.

Our methodology consists of the steps $1 \div 5$ below 4 . Steps $3 \div 4$ are inherited from [11].

1. The architecture (say $G$ ) corresponding to the configuration of the current system is computed using the tracking tree $T$ and the corresponding environment $\mathscr{T}$ of the system.

2. Identify the sub-tree $T_{R}$ of $T\left(t^{\prime} \bowtie T_{R}\right)$ that corresponds to the RHS term $t^{\prime}$ of the reconfiguration applied.

3. Check whether $G$ satisfies $\phi_{\text {inv }}\left(G \models \phi_{\text {inv }}\right)$.

(a) If $G=\phi_{\text {inv }}$ then the style is not violated.

(b) If $G \not \models \phi_{\text {inv }}$ then go to step 4

4. For each production $p$, compute the weakest pre-condition $\psi$ with respect to $\phi_{\text {inv }}$.

(a) Select a production $p: L \rightarrow R$ and let $\sigma: L \rightarrow G$ be the morphism from $L$ to $G$ such that $G \backslash \sigma\left(E_{L}\right) \models \psi$ (if any); apply $p$ to $G$ to determine the reconfiguration needed for the system to reach a valid state.

(b) If the designer considers not satisfactory the reconfigured system obtained in the previous stage or if there is no production $p$ such that $G \backslash \sigma\left(E_{L}\right) \models \psi$, then the designer may either, repeat step 4 a by replacing $G$ with $G \backslash \sigma\left(E_{L}\right)$ and $\phi_{\text {inv }}$ with $\psi$ or, try step 5

5. Given $T_{R}$ computed in step 2 the designer can select a 2-tier subtree $T_{R}^{\prime}$ of $T_{R}$ that contains a parent vertex and all its leaf children correspond to replaceable edges through the environment $\mathscr{T}$. Using $T_{R}^{\prime}$ in Definition 12 we parse the graph as defined below and repeat step 4.

Definition 12 (Parsing) Given a graph $G$, a corresponding 2-tier sub-tree $T$ with root $\diamond$ and an environment $\mathscr{T} . \quad p: L \stackrel{i}{\rightarrow} R$ is the production returned from $\mathscr{T}^{(2)}(\diamond)$. Given an instance $L^{\prime}$ of $L$ through the isomorphism $\imath: L \rightarrow L^{\prime}$ and let $\sigma^{\prime}: R \rightarrow G$ a graph $G^{\prime}=G \backslash\left(E_{G} \cup \sigma^{\prime}\left(R^{\circ}\right)\right) \cup L^{\prime \prime}\left(R^{\circ}\right.$ refer to the internal nodes of $R)$ is the graph obtained by parsing $G$ with $p$ under the morphism $\sigma^{\prime}$ iff $L^{\prime \prime}=L^{\prime}[l(l) \mapsto$ $\left.\sigma^{\prime}(i(l)) \mid l \in \operatorname{Im}(i)\right]$ and there exist no edge in $G$ that is non-replaceable.

Identifying the architecture of a system is non-deterministic in the original specification of ADR (cf. [4]). Using our approach we can identify and retrieve the architecture of the system with all the information of how it has been adapted so far using our monitoring mechanism. This mechanism is used in step 1 for retrieving graph $G$ and in step 2 for identifying the subtree of the tracking tree that has been reconfigured. In step 3 , we assume that an underlying monitoring mechanism uses the $=$ relation of our logic to determine if the graph $G$ identified in step 1 violates the invariant. In such case, step 4 uses the weakest pre-condition algorithm [11] on each production to compute their weakest pre-conditions (this step does not need to be re-iterated at each reconfiguration but instead all the weakest pre-conditions can be computed offline and reused accordingly). In step 4a, if the graph representing the violated system satisfies one of the computed weakest pre-conditions then the corresponding production is a candidate to

\footnotetext{
${ }^{4}$ Recall that the designer has to specify productions and the architectural invariant $\phi_{\text {inv }}$ so to establish the architectural style of interest (as done in Example 16.
} 
re-establish the architectural style and trigger the appropriate reconfigurations on the invalid system. In step $4 \mathrm{~b}$ the designer has to decide whether to stop or continue the process. In the latter case, the idea is to repeat steps 4 and 4 a replacing $G$ with $G \backslash \sigma\left(E_{L}\right)$ and $\phi_{\text {inv }}$ with $\psi$ so to compute the weakest pre-condition of the weakest pre-condition computed in the previous iteration. This, allows us to exploit every possible sequence of productions that can be applied in order to enforce the architectural style. Note that the morphism that invalidates $G=\phi_{\text {inv }}$ indicates which part of the system has to be rewritten, while the production $p$ suggests plausible reconfigurations. If the above steps do not prove to be satisfactory then in step 5 the designer uses the tree computed in step 2 and selects which branch of the tree can be abstracted using the parsing mechanism in Definition 12 . After parsing the graph we repeat steps $4 \div 5$ until either, a possible sequence of productions is identified that can potentially fix the architectural style, or, the designer decides to stop the process.

\section{Conclusion}

In this paper we defined a framework that allows us to exploit the "hierarchical nature" of ADR graphs. In particular, we used the functional reading of ADR productions as well as its reconfigurations that preserve the identity of components throughout the rewriting. Our framework permits to tackle certain architectural aspects of the design and allows the designer to identify and address problems at the architectural level.

Also, we refine the methodology proposed in [11] to automatically compute possible reconfigurations that recover from architectural style violations. Our refinement iteratively computes the weakest precondition to find a possible sequence of reconfigurations (if any) that re-establishes the architectural style of the system.

In this paper we focused on the development of the technical presentation of our framework. We proposed a monitoring mechanism through which the evolution of a computation is recorded and maintained in a tree-like structure reflecting the hierarchical nature of ADR graphs. We then exploit this mechanism to formally define more efficient parsing algorithms as well as more efficient ways of applying reconfigurations. Interestingly, this approach brings forth the definition of a new rewriting mechanism for ADR reconfiguration rules. More precisely, instead of parsing an ADR graph searching for a sub-graph matching the RHS of a reconfiguration rule, we propose a rewriting mechanism that visits the trees describing the graph evolution to find the match. We argue that this is more efficient than parsing the graph at the negligible cost of recording the evolution of the system through the monitoring. One could argue that the run-time monitoring of the system could be inefficient. In this respect, we note that a form of monitoring is necessary when dealing with self-configuring or self-healing systems; quoting [9] "autonomic system might continually monitor its own use". Since autonomic systems are the reference systems of this work, and, as a matter of fact, a form of monitoring is indispensable in order to identify run-time violation of the style, we argue that our approach simply adds to the necessary monitoring activities the cost of tracking the evolution of systems.

This paper completes and refines the framework initially proposed in [11]. We contemplate a new research direction to devise autonomic systems where component managers use architectural elements in the reconfigurations they distill. From this point of view, ADR is particularly suitable due to the fact that it can represent not only architectural level aspects of systems, but it can also be used to represent operational semantics by e.g. encodings of process calculi or modelling languages [3, 2]. This would immediately establish a connection between $\mathrm{DbC}$ approaches of abstractions levels close to the implementation of systems (for instance, see [1, 10]) with the DbC approach for ADR suggested in [11]. 
We conclude by commenting about the linearity conditions imposed on the reconfigurations rules (c.f. (6) and Definition 11). The linearity of the LHS of a reconfiguration rule can be relaxed at the cost of making the semantic of the matching more complex since multiple occurrences of the same variable would account for checking the existence of an isomorphism among different subgraphs. Instead, the linearity condition on the RHS of a production can be relaxed by simply using the counterpart semantic mechanism described in [5] to keep track of one copy of the variable. Finally, we note that in [4], reconfigurations rules of the form $r(x): p(y) \rightarrow q(x, y)$ are considered, where $x$ act as a parameter of the rule that can be used in its LHS or RHS. Such rules can be easily added to our framework using Definition 11 by mapping $\eta(x)$ to the fresh input graph.

\section{References}

[1] Laura Bocchi, Kohei Honda, Emilio Tuosto \& Nobuko Yoshida (2010): A Theory of Design-by-Contract for Distributed Multiparty Interactions. In: CONCUR, pp. 162-176, doi:10.1007/978-3-642-15375-4_12.

[2] Roberto Bruni, Howard Foster, Alberto Lluch-Lafuente, Ugo Montanari \& Emilio Tuosto (2011): A Formal Support to Business and Architectural Design for Service-Oriented Systems. In Martin Wirsing \& Matthias M. Hölzl, editors: Results of the SENSORIA Project, Lecture Notes in Computer Science 6582, Springer, pp. 133-152, doi:10.1007/978-3-642-20401-2_7.

[3] Roberto Bruni, Fabio Gadducci \& Alberto Lluch Lafuente (2010): An Algebra of Hierarchical Graphs and its Application to Structural Encoding. Scientific Annals of Computer Science 20, pp. 53-96.

[4] Roberto Bruni, Alberto Lluch-Lafuente, Ugo Montanari \& Emilio Tuosto (2008): Style-Based Architectural Reconfigurations. In: Bulletin of the EATCS, pp. 161-180.

[5] Fabio Gadducci, Alberto Lluch-Lafuente \& Andrea Vandin (2012): Counterpart Semantics for a SecondOrder Calculus. Fundam. Inform. 118(1-2), pp. 177-205, doi:10.3233/FI-2012-709.

[6] Dina Goldin, Scott A. Smolka \& Peter Wegner, editors (2006): Interactive Computation. Springer-Verlag.

[7] Markus C. Huebscher \& Julie A. McCann (2008): A survey of autonomic computing - degrees, models, and applications. ACM Comput. Surv. 40(3), pp. 7:1-7:28, doi $10.1145 / 1380584.1380585$.

[8] Bart Jacob, Richard Lanyon-Hogg, Devaprasad K. Nadgir \& Amr F. Yassin (2004): A Practical Guide to the IIBM Autonomic Computing Toolkit.

[9] Jeffrey O. Kephart \& David M. Chess (2003): The Vision of Autonomic Computing. Computer 36(1), pp. 41-50, doi:10.1109/MC.2003.1160055

[10] Yi Liu \& H. Conrad Cunningham (2002): Software Component Specification Using Design by Contract. In: Proceeding of the South East Software Engineering Conference.

[11] Kyriakos Poyias \& Emilio Tuosto (2012): Enforcing Architectural Styles in Presence of Unexpected Distributed Reconfigurations. In: ICE, pp. 67-82, doi:10.4204/EPTCS.104.7.

[12] Richard N. Taylor, Nenad Medvidović \& Eric M. Dashofy (2009): Software Architecture: Foundations, Theory, and Practice. John Wiley and Sons, doi $10.1145 / 1810295.1810435$. 